\title{
Comparative Analysis of Streptococcus Agalactiae S03 and S07 Isolated From Schizothorax Prenanti With Different Antibiotic Resistance and Virulence
}

\section{Lishuang Deng}

Sichuan Agricultural University

\section{Yajun Li}

Sichuan Agricultural University

Yi Geng ( $\nabla$ gengyisicau@126.com )

Sichuan Agricultural University

\section{Defang Chen}

Sichuan Agricultural University

\section{Ping Ouyang}

Sichuan Agricultural University

\section{Xiaoli Huang}

Sichuan Agricultural University

Hongrui Guo

Sichuan Agricultural University

\section{Zhicai Zuo}

Sichuan Agricultural University

\section{Chao Huang}

Sichuan Agricultural University

\section{Zhengli Chen}

Sichuan Agricultural University

\section{Weiming Lai}

Sichuan Agricultural University

\section{Tayyab Rehman}

Sichuan Agricultural University

\section{Research Article}

Keywords: Streptococcus agalactiae, whole genome sequencing, antibiotic resistance, virulence, pathogenicity analysis, Schizothorax prenanti

Posted Date: April 7th, 2021 
DOl: https://doi.org/10.21203/rs.3.rs-390950/v1

License: (c) (1) This work is licensed under a Creative Commons Attribution 4.0 International License. Read Full License 


\section{Abstract}

Streptococcus agalactiae(GBS), is an important Gram-positive pathogen of fish aquaculture worldwide. In this study, we performed a comparative analysis of GBS S03 and S07 isolated from Schizothorax prenanti and explored the association between phenotypic antibiotic resistance as well as virulence and the genomic characteristics. Antimicrobial sensitivity tests on 12 common antibiotics using the disc diffusion method revealed that the S03 showed resistance to seven antibiotics, while S07 showed sensitivity to the tested antibiotics. Pathogenicity analysis demonstrated greater virulence of S07 than S03. Then, the occurrence of antibiotic resistance and virulence genes was identified using wholegenome sequence (WGS) of S03 and S07. There were mefE, tetO, InuB, IsaE, APH3', and sat-4 resistance genes present only in $\mathrm{S} 03$ genome. And just $\mathrm{S} 03$ had gyrA and parC genes mutations. In addition to 51 virulence genes in both $\mathrm{S} 03$ and $\mathrm{S} 07$ genomes, $\mathrm{S} 07$ additionally carried virulence genes associated with invasion, such as $S A N \_1519$, rfb $A$ and $c y / E$ genes. There was complete concordance between genotypic evidence and phenotypic characteristics. Virulence factors and phylogenetic analysis showed that S03 and human sources shared an extremely close evolutionary relationship. Our findings provide important proof for using WGS as an effective tool of phenotypic predictions of GBS.

\section{Introduction}

Streptococcus agalactiae, or group B streptococcus (GBS), is an important Gram-positive pathogen responsible for morbidity and mortality in fish aquaculture worldwide (Evans et al. 2008; Chong et al. 2016; Kannika et al. 2017). The GBS is also an emerging pathogen associated with neonatal meningitis in humans, mastitis in cows and hematosepsis in rabbits (Bisharat et al. 2004; Ren et al. 2013; Veeraraghavan et al. 2017). To date, GBS can be classified into 10 serotypes (la, lb, and II to IX), of these serotype la, Ib and III are regarded as the most predominant in fish GBS infection (Slotved et al. 2007; Li et al. 2013). In fish farms, transmission of GBS is possible through direct contact among individuals with cohabitation or indirectly through immersion in contaminated water of culture systems (Facimoto et al. 2017).

Whole-genome sequencing (WGS) technology has become a fast and affordable tool that is affecting research in the fields of genetics, microbiology, and ecology, as well as public health surveillance and response (Zhao et al. 2016). WGS captures the full extent of bacterial genomic diversification and allows for genome wide comparisons of clinical isolates with each other (Lee et al. 2015). Comparative genome analysis between bacterial strains which are greatly different in host specificity or virulence may help to rapidly screen for dispensable genes, gene deletions or mutations, and differentially-expressed proteins (Wang et al. 2017). Thus, WGS is an effective way of studying the mechanisms of cross-host infection, immunogenicity, pathogenicity, and resistance genotypes of bacteria. At present, hundreds of complete genomes of GBS have been sequenced. However, our understanding of the piscine GBS at the whole genome level is currently limited. Although some complete genome sequences and draft genome sequences of piscine GBS isolates have been recorded so far (Areechon et al. 2016; Facimoto et al. 2017; Liu et al. 2012; Liu et al. 2013; Pridgeon and Zhang 2014; Jaglarz et al. 2018), the correlation between 
WGS analysis and phenotypic characteristics has not been reported. Here, this is the first report on draft genomes of GBS S03 and S07 isolated from Schizothorax prenanti with different pathogenicity and drugresistance. Draft genomes of GBS S03 and S07 are important additions to the GenBank database of piscine GBS.

In the current study, antimicrobial susceptibility testing, experimental infection and WGS analysis were carried out to compare two GBS clinical strains isolated from Schizothorax prenanti. Phylogenetic analysis was performed to identify their evolutionary relationships in GBS. We attempted to assess the use of WGS to evaluate the genomic diversity and genotypic prediction of drug-resistant phenotype and pathogenicity of them.

\section{Materials And Methods \\ 2.1. Bacterial strains}

GBS S03 and S07 were isolated from diseased Schizothorax prenanti, with characteristic clinical and pathogenic meningoencephalitis from the culture collection of the College of Veterinary Medicine, Sichuan Agricultural University, China. Serotyping assigned GBS S03 to serotype III and S07 to serotype la by cps cluster.

\subsection{Antimicrobial susceptibility tests}

Antimicrobial susceptibility to vancomycin (VAN), penicillin (PEN), cephalexin (CFX), enrofloxacin (ENR), ofloxacin (OFX), norfloxacin (NOR), tetracycline (TET), doxycycline (DOX), erythromycin (ERY), gentamicin (GEN), florfenicol (FLO) and clindamycin (CLIN) was performed by the disc diffusion method.

Antimicrobial susceptibility tests results were interpreted according to Clinical and Laboratory Standards Institute (CLSI) breakpoints. Escherichia coli (ATCC 25922) was used as the reference strain for quality control according to CLSI guidelines.

\subsection{Pathogenicity experiments}

To confirm the pathogenicity and virulence of GBS S03 and S07, we injected the two strain suspensions into healthy Schizothorax prenanti and Danio rerio, intraperitoneally. Healthy Schizothorax prenanti (11.5 $\pm 1.2 \mathrm{~g})$ and Danio rerio $(0.4 \pm 0.05 \mathrm{~g})$ were purchased from Sichuan Ya-fish Company and Model Animal Research Center Of Nanjing University, respectively. 260 Schizothorax prenanti and Danio rerio were kept in 13 groups equally, respectively, in 120-L plastic tanks ( $n=20 /$ tank or group) with aeration and were acclimatized at $24 \pm 1^{\circ} \mathrm{C}$ for 7 days. The Schizothorax prenanti of 6 experiment groups were challenged with $0.1 \mathrm{ml}$ of bacterial suspensions of the $\mathrm{SO} 3$ and the other 6 groups were injected with the same volume of S07 suspensions in sterile $0.8 \% \mathrm{NaCl}$ solution at concentrations of $1.0 \times 10^{4}, 1.0 \times 10^{5}, 1.0 \times 10^{6}$, $1.0 \times 10^{7}, 1.0 \times 10^{8}, 1.0 \times 10^{9} \mathrm{cfu} \cdot \mathrm{ml}^{-1}$. The control fish $(\mathrm{n}=20)$ were injected with $0.1 \mathrm{ml} 0.8 \% \mathrm{NaCl}$ solution. Similarly, the Danio rerio of test groups were injected intraperitoneally with $0.05 \mathrm{~mL}$ of bacterial suspensions of the $\mathrm{S} 03$ and $\mathrm{S} 07$ in sterile $0.8 \% \mathrm{NaCl}$ solution at different concentrations mentioned 
above. The control animals were injected with $0.05 \mathrm{~mL} 0.8 \% \mathrm{NaCl}$ solution. Mortality was recorded daily for $21 \mathrm{~d}$ and mean lethal dose $\left(\mathrm{LD}_{50}\right)$ values were calculated. All the fish were euthanized by using 300 $\mathrm{mg} \cdot \mathrm{L}^{-1}$ MS222 (yuanye Bio. Co. Ltd., Shanghai, China) for $21 \mathrm{~d}$.

\subsection{Whole genome sequencing and annotation}

To explore further differences between S03 and S07, their draft genomes were sequenced and analyzed at whole genome level. The genomic DNA of the two strains were extracted using the TIANamp bacteria DNA kit (Tiangen, Beijing, China) and applied for library preparation using the Nextera DNA sample preparation kit (Illumina, San Diego, USA). The samples were accessed for the genome sequencing on an Illumina HiSeqTM 2000 (Illumina Inc., San Diego, USA) using a paired-end 2×100 bp protocol. The whole genome sequences were assembled using CLC Genomics Workbench 10.0 software (QIAGEN, Hilden, Germany). Contigs were initially annotated using Rapid Annotations using Subsystems Technology (RAST) and then manually checked. Resistance-related genes of the two strains were analyzed using ResFinder 2.1 server (Zankari et al. 2012). Gene clusters were classified into COG (Cluster of Orthologous Groups of proteins) categories (http://www.ncbi nlm.nih.gov/COG/) according to BLAST annotations.

\subsection{Identification of antibiotic resistance genes and virulence factors}

To find antibiotic-resistant genes in two GBS strains genomes, known antibiotic resistance genes were downloaded from the Comprehensive Antibiotic Resistance Database (CARD) and aligned with all coding sequences (CDSs) of those genomes using BLAST (https://card.mcmaster.ca/). A similar method has been applied to identify virulence genes from the Virulence Factor Database (VFDB) (http://www.mgc.ac.cn/VFs/).

\subsection{Phylogenetic tree}

A phylogenetic tree of GBS strains was constructed based on seven housekeeping genes (adhP $[$ pheSlatrl $g / n A \square s d h A \square g l c K$ and $t k t)$ as previously described (Jones et al. 2003). The alignments of these genes were concatenated into a single sequence alignment and a maximum likelihood tree was reconstructed using MEGA (version 10.0). The allele number and sequence type (ST) were assigned by multilocus sequence typing (MLST) website (https://pubmlst.org/organisms/streptococcus-agalactiae).

\section{Results}

\subsection{General features of the GBS S03 and S07 genomes}

Characteristics of the two genomes are summarized in Table 1.The circular maps of two strains showed that the genome of S03 was 2,156,979 bp in length (Fig. S1a), while that of S07 was 1,790,578 bp in length (Fig. S1b). Genome sequence of both strains showed 35\% G + C contents. RAST server predicted the 2,160 and 1,641 CDSs for S03 and S07 strains, respectively. On the basis of COG classification of S03 
and $\mathrm{S} 07,23.7 \%$ and $22.6 \%$ of CDSs were responsible for information storage and processing, whereas $37.5 \%$ and $39.4 \%$ were for metabolism (Fig. S2).

Table 1

General features of GBS S03 and S07.

\begin{tabular}{|lll|}
\hline Features & S03 & S07 \\
\hline Host & $\begin{array}{l}\text { Schizothorax } \\
\text { prenanti }\end{array}$ & $\begin{array}{l}\text { Schizothorax } \\
\text { prenanti }\end{array}$ \\
\hline Sequence type & ST19 & ST891 \\
\hline Serotyping & III & la \\
\hline Genome size (bp) & $2,156,979$ & $1,790,578$ \\
\hline G + C content (\%) & 35 & 35 \\
\hline Total CDSs & 2,160 & 1,641 \\
\hline $\begin{array}{l}\text { CDSs responsible for information storage and } \\
\text { processing (\%) }\end{array}$ & 23.7 & 22.6 \\
\hline CDSs responsible for metabolism (\%) & 37.5 & 39.4 \\
\hline Transfer RNA & 42 & 29 \\
\hline
\end{tabular}

The genome sequences of S03 and S07 have been deposited in the NCBI GenBank database under the accession numbers NAPX00000000 and NHZY00000000, respectively.

\subsection{Antibiotic resistance profile and antibiotics resistance genes}

Antimicrobial susceptibility distribution of S03 and S07 was given in Fig. 1a. The results showed that there were significant differences in resistance between the two strains. Antimicrobial tests revealed that S03 showed multidrug resistance against OFX, NOR, ENR, TET, DOX, ERY and CLIN (Table S1). By contrast, S07 was sensitive or intermediate to all 12 tested antibiotics.

The genomes of GBS S03 and S07 showed that S03 had 20 resistance genes, while S07 had 14 resistance genes (Fig. $1 \mathrm{~b}$ and Table S2). In addition to two macrolide resistant genes (macB and ermB) and one tetracycline resistant gene tetM, S03 also had mefE and tetO genes. Meanwhile, S03 had additionally InuB, IsaE, $A P H 3$ ', and sat-4 genes not present in S07. Both strains carried genes encoding resistance to fluoroquinolone: parC, gyrA, gyrB, norA and norB, however, just S03 had chromosomal gyrA [81:S-L] and $\operatorname{parC}$ [79:S-Y] mutations (Fig. S3).

\subsection{Pathogenicity experiments and virulence genes}


At 21 days post-infection (dpi), GBS S07 induced higher levels of mortality and revealed a greater virulence than S03 in the pathogenicity experiments (Fig. 2). Concretely, The LD $\mathrm{L}_{50}$ value of S03 for Schizothorax prenanti and Danio rerio was $5.27 \times 10^{8} \mathrm{cfu} \cdot \mathrm{ml}^{-1}$ and $7.09 \times 10^{7} \mathrm{cfu} \cdot \mathrm{ml}^{-1}$ respectively, while that of S07 was $8.9 \times 10^{3} \mathrm{cfu} \cdot \mathrm{ml}^{-1}$ for Schizothorax prenanti and $1.88 \times 10^{3} \mathrm{cfu} \cdot \mathrm{ml}^{-1}$ for Danio rerio. S03 strain caused $25 \%$ and $60 \%$ mortality in Schizothorax prenanti at the $1.0 \times 10^{8}$ and $1.0 \times 10^{9} \mathrm{cfu} \cdot \mathrm{ml}^{-1}$, while S07 caused $55 \%, 75 \%, 95 \%, 100 \%, 100 \%$ and $100 \%$ at $1.0 \times 10^{4}, 1.0 \times 10^{5}, 1.0 \times 10^{6}, 1.0 \times 10^{7}, 1.0 \times 10^{8}$ and $1.0 \times 10^{9} \mathrm{cfu} \cdot \mathrm{ml}^{-1}$ concentrations respectively. Danio rerio had $20 \%, 35 \%, 55 \%$ and $70 \%$ cumulative mortality caused by S03 at $1.0 \times 10^{6}, 1.0 \times 10^{7}, 1.0 \times 10^{8}, 1.0 \times 10^{9} \mathrm{cfu} \cdot \mathrm{ml}^{-1}$, while caused by S07 were $70 \%$, $85 \%, 95 \%, 100 \%, 100 \%$ and $100 \%$ at $1.0 \times 10^{4}, 1.0 \times 10^{5}, 1.0 \times 10^{6}, 1.0 \times 10^{7}, 1.0 \times 10^{8}, 1.0 \times 10^{9} \mathrm{cfu} \cdot \mathrm{ml}^{-1}$ respectively. There was no mortality in the control groups during the observation period.

Fifty-one virulence genes were identified in the both genomes (Table 2). Among these, bibA, dltA, fbsA, pavA, psaA, srtA, fbsB, pavB, lap, lep, Imb and srr-1 were involved in attachment; CLL_A 2400, hylB, Igt, $m f 3$, and sugC were involved in invasion; $c f b, c p s A-L, c p s A-Y$, manA, neuA-D, uppS, rgpA, rmIA, scpA, rgpB, scpB, oppF, rgpG, sip, BC5263, EFD32, EFD32_0765 and SMU.322c were involved in evading/destroying host defenses; and other virulence genes, included were $\operatorname{coxK} 2, c l p C, c / p E, c l p P, c p p A, \operatorname{ms} b A$, nanA, gbpB, fhuC, licD, lisR, IpIA1, groEL, htrA/deg, CT396 and stp. Comparison of the virulence genes between $\mathrm{S03}$ and S07 showed differences. S03 carried SAG1404-1408, rib, SAK_0778-0779 and gbs0628-0629 associated with attachment genes, while S07 were carrying $S A N \_1519$, rfbA and cylE involved in invasion genes.

Table 2

Virulence genes of GBS S03 and S07.

\begin{tabular}{|c|c|c|}
\hline Class & S03 & S07 \\
\hline Attachment & $\begin{array}{l}\text { bibA, dltA, fbsA, pavA, psaA, srtA, fbsB, } \\
\text { pavB, Iap, lep, Imb, srr- } 1 \text {, rib, SAG1404, } \\
\text { SAG1405, SAG1406, SAG1407, SAG1408, } \\
\text { SAK_0778, SAK_0779, gbs0628, gbs0629 }\end{array}$ & $\begin{array}{l}\text { bibA, dltA, fbsA, pavA, psaA, sitA, } \\
\text { fbsB, pavB, lap, lep, Imb, srr-1 }\end{array}$ \\
\hline Invasion & CLL_A 2400, hylB, Igt, mf3, sugC & $\begin{array}{l}\text { CLL_A 2400, hylB, Igt, mf3, sugC, } \\
\text { SAN_1519, rfbA, cylE }\end{array}$ \\
\hline $\begin{array}{l}\text { Evading/destroying } \\
\text { host defenses }\end{array}$ & $\begin{array}{l}\text { Cfb, cpsA-L, cpsA-Y, manA, neuA-D, uppS, } \\
\text { rgpA, rmIA, scpA, rgpB, scpB, oppF, rgpG } \\
\text { sip, BC5263, EFD32, EFD32_0765, } \\
\text { SMU.322c }\end{array}$ & $\begin{array}{l}\text { Cfb, cpsA-L, cpsA-Y, manA, neuA- } \\
D \text {, uppS, rgpA, rmIA, scpA, rgpB, } \\
\text { scpB, oppF, rgpG, sip, BC5263, } \\
\text { EFD32, EFD32_0765, SMU.322c }\end{array}$ \\
\hline $\begin{array}{l}\text { Other virulence } \\
\text { genes }\end{array}$ & $\begin{array}{l}\text { coxK2, clpC, clpE, clpP, cppA, msbA, nanA, } \\
\text { gbpB, fhuC, licD, lisR, IplA1, groEL, } \\
\text { htrA/deg, CT396, stp }\end{array}$ & $\begin{array}{l}\text { coxK2, clpC, clpE, clpP, cppA, } \\
\text { msbA, nanA, gbpB, fhuC, licD, } \\
\text { lisR, IplA1, groEL, htrA/deg, } \\
\text { CT396, stp }\end{array}$ \\
\hline
\end{tabular}

\subsection{Phylogenetic analysis}


By MLST, S03 was determined to be ST19, while S07 was ST891. To investigate the phylogenetic relationships of our two isolates to other GBS strains, we used the maximum likelihood method to construct a phylogenetic tree (Fig. 3). Phylogenetic analysis was performed using 38 reported GBS strains. Interestingly, S03 was closely related to Sag158, H002 and SG-M25 strains isolated from human while $\mathrm{S} 07$ was grouped separately.

\section{Discussion}

Since 2008, GBS has been present and emerging in tilapia in China (Zhang et al. 2008). Liu et al. (2013) reported the first complete genome sequence of the piscine GBS strain isolated from cultured tilapia in China. In this study, draft genomes of GBS S03 and S07 isolated from Schizothorax prenanti have been recorded. The genome size of $\mathrm{S} 07$ was approximately $1.79 \mathrm{Mbp}$ and approximately $0.37 \mathrm{Mbp}$ smaller than S03. There were more predicted CDSs in the S03 genome than in S07. Genome size is the result of a balance between amplification and loss of DNA, which evolves toward an optimal state for organismal fitness (Devos et al. 2002; Lynch 2006). Furthermore, genomic changes leading to streamlined genomes with increased pathogenicity and virulence are important (Siguier et al. 2014). They might involve fragment recombination and contribute to the different virulence of S03 and S07.

Antimicrobial tests revealed that the GBS S03, showed multidrug resistance, but S07, showed drugsensitive to the tested antibiotics. The differences in drug resistance between the two strains were significant, which may have been associated with environmental variability and/or the frequent and abusive use of chemotherapy in aquaculture (Abuseliana et al. 2010; Florindo et al. 2010). In the current study, the phenotypes of two strains were in concordance with genomic characteristics revealed by WGS. Two strains had fluoroquinolone genes: parC, gyrA, gyrB, norA and norB, however, just $\mathrm{SO} 3 \mathrm{had}$ chromosomal gyrA [81:S-L] and parC [79:S-Y] mutations, explaining high-level fluoroquinolone resistance (Kawamura et al. 2003; Salma et al. 2013; Doumith et al. 2017). In addition to two macrolide resistant genes ( $m a c B$ and $e r m B$ ) and one tetracycline resistant gene tetM, S03 additionally had mefE and tetO, which contributed to high-level macrolide and tetracycline resistance (Poyart et al. 2003; Doumith et al. 2017), as observed in this study. These results are consistent with what we have previously found through traditional methods (Deng et al. 2019), but WGS is clearly more efficient and accurate. Meanwhile, GBS S03 had additional InuB, IsaE, APH3', and sat-4 genes not present in $\mathrm{S} 07$ strain, the first two of which play an important regulatory role in lincosamide resistance (Faccone et al. 2010). The combination of $A P H 3^{\prime}$ and sat-4, associated with transposon Tn 5405 among Gram-positive bacteria, encodes resistance to aminoglycosides (except gentamicin) and streptothricins (Derbise et al. 1997; Werner et al. 2003). These results reveal a trend towards concordance between resistance phenotype and genotypic evidence for antibiotic resistance.

The pathogenicity of the two strains were compared by intraperitoneal injection in Schizothorax prenanti or Danio rerio, which demonstrated that S07 showed much greater virulence than S03. S07 also carried more virulence genes associated with invasion, such as $S A N \_1519$, IfbA and cylE genes. However, it was counter to the previous report on GBS from Tilapia in Thailand (Areechon et al. 2016), which is most likely 
related to the diversity in host, area and strain. Fifty one virulence genes in both GBS S03 and S07 involved in attachment, persistence, evading host defenses, tissue penetration, and toxin-mediated diseases had been identified in the GBS genomes, providing evidence of the pathogenicity of GBS (Godoy et al. 2013; Chen et al. 2014; Kayansamruaj et al. 2014). Differences in virulence genes between the two strains are mainly reflected in the genes encoding pili, including SAG_1404-1408, gbs0628-0629 and SAN_1519. Genes encoding pili in GBS are located within two distinct loci, denoted pilus islands 1 and 2 (PI-1 and PI-2), and comparative analysis of available genomes revealed two variants of PI-2, designated PI-2a and PI-2b (Rosini et al. 2006). Gbs0628-0629 genes, SAG_1404-1408 genes, and SAN_1519 gene are located in PI-1, PI-2a and PI-2b, respectively (Rosini et al. 2006; Nobbs et al. 2008). Pili are supposed to be important virulence factors (Nobbs et al. 2008). In GBS, pilus 1 is related to the immune escape, pilus $2 a$ confers formation of biofilms, and pilus $2 b$ is linked to the ability of attachment and invasion (Rinaudo et al. 2010; Jiang et al. 2012). In this study, S03 carried PI-1 plus PI-2a type pilus islands while S07 carried PI-2b type pilus island, which suggested there was concordance between pathogenicity and WGS result. Previous studies showed that most of the fish-sources strains carried PI-2a type, PI-2b type or $\mathrm{PI}-1$ plus $\mathrm{PI}-2 \mathrm{~b}$ type pilus islands, while in human strains, a great number of GBS carried $\mathrm{PI}-1$ plus $\mathrm{PI}-2 \mathrm{a}$ type or PI-1 plus PI-2b type (He et al. 2017).

Interestingly, phylogenetic analysis showed that S03 was closely related to human sources (Sag158, H002 and SG-M25) while S07 was grouped separately. Combined with genome size and phenotypic characteristics, we hypothesized that they had different origins and evolutionary directions. Further, bestowed pili types and phylogenetic analysis, we hypothesized that $\mathrm{S} 03$ had a common source with human GBS or the possibility of cross-infection with human strains. Unfortunately, we currently do not have adequate epidemiological information to confirm this hypothesis. Although the knowledge about the ability of GBS to cross the interspecies barrier and allow human-derived strains to infect animals, or vice versa, is poorly understood, Pereira et al. (2010) demonstrated that GBS strains from human and bovine origins could infect fish. Rajendram et al. (2016) found that human could be infected by fishisolated GBS by consuming of raw fish. Therefore, we should attach great importance to cross-host transmission of GBS and its potential threat to public health security. In addition, whether our two strains in this study will exchange and transfer resistance genes and virulence genes in nature, with the appearance of new strains with great toxicity and extreme drug resistance, which deserve further research and attention.

\section{Conclusions}

In this study, GBS S03 and S07 isolated from Schizothorax prenanti showed greatly distinct phenotypic characteristics, including drug-resistant phenotype and pathogenicity, and they were diverse at the genome level. There was finished concordance between genotypic evidence and phenotypic characteristics. Our findings provide important proof for using WGS as an operational tool of phenotypic predictions of GBS. 


\section{Abbreviations}

GBS: Streptococcus agalactiae; WGS: Whole genome sequencing; LD50: mean lethal dose; CLIN, clindamycin; ERY, erythromycin; ENR, enrofloxacin; NOR, norfloxacin; OFX, ofloxacin; TET, tetracycline; DOX, doxycycline; PEN, penicillin; CFX, cephalexin; VAN, vancomycin; GEN, gentamicin; FLO, florfenicol; dpi: days post-infection; ARDB: Antibiotic Resistance Genes Database; VFDB: Virulence Factor Database

\section{Declarations}

\section{Ethics approval and consent to participate}

All animal procedures were conducted in accordance with Animal Experiment General Requirement in China (record number GB/T 35823 - 2018) and were approved by the Institutional Animal Care and Use Committee (IACUC) of Sichuan Agricultural University.

\section{Consent for publication}

Not applicable.

\section{Availability of data and materials}

All data generated or analyzed during this study are included in this article.

\section{Competing interests}

The authors have declared that no competing interests exist.

\section{Funding}

This work was supported by the Sichuan Key Research and Development Project (No 2018NZ0007). The funders had no role in the study design, data collection and analysis, decision to publish, or preparation of the manuscript.

\section{Authors' contributions}

Conceptualization, Lishuang Deng, Yajun Li, and Yi Geng; Data curation, Yajun Li; Formal analysis, Lishuang Deng, Yajun Li, and Yi Geng; Methodology, Lishuang Deng, Yajun Li, and Yi Geng; Supervision, Defang Chen, Yangping Ou, Xiaoli Huang, Hongrui Guo, Zhicai Zuo, Chao Huang, Zhengli Chen and Weiming Lai; Validation, Yi Geng; Writing-original draft, Lishuang Deng; Writing-review \& editing, Lishuang Deng, Yi Geng, and Tayyab Rehman. All authors read and approved the final manuscript.

\section{Acknowledgments}

We gratefully acknowledge members of Fish Disease Research Center, Sichuan Agricultural University. 


\section{References}

1. Abuseliana A, Daud H, Aziz SA, Bejo SK, Alsaid M (2010) Streptococcus agalactiae the Etiological Agent of Mass Mortality in Farmed Red Tilapia (Oreochromis sp.). Journal of Animal and Veterinary Advances 9: 2640-2646. https://doi.org/10.3923/javaa.2010.2640.2646

2. Areechon N, Kannika K, Hirono I, Kondo H, Unajak S (2016) Draft Genome Sequences of Streptococcus agalactiae Serotype la and III Isolates from Tilapia Farms in Thailand. Genome Announc 4: e00122-00116. https://doi.org/10.1128/genomea.00122-16

3. Bisharat N, Crook DW, Leigh J, Harding RM, Ward PN, Coffey TJ, Maiden MC, Peto T, Jones N (2004) Hyperinvasive neonatal group B streptococcus has arisen from a bovine ancestor. Journal of clinical microbiology 42: 2161-2167. https://doi.org/10.1128/jcm.42.5.2161-2167.2004

4. Chen H, Yang X, Wang Q, Zhao C, Li H, He W, Wang X, Zhang F, Wang Z, Chen M, Zhu B, Wang H (2014) Insights on evolution of virulence and resistance from the whole-genome analysis of a predominant methicillin-resistant Staphylococcus aureus clone sequence type 239 in China. Chinese Science Bulletin 59: 1104-1112. https://doi.org/10.1007/s11434-014-0149-1

5. Chong SM, Wong WK, Lee WY, Tan ZB, Tay YH, Teo XH, Chee LD, Fernandez CJ (2016) Streptococcus agalactiae outbreaks in cultured golden pomfret, Trachinotus blochii (Lacépède), in Singapore. Journal of fish diseases 40: 971-974. https://doi.org/10.1111/jfd.12570

6. Deng LS, Li YJ, Geng Y, et al. (2019) Molecular serotyping and antimicrobial susceptibility of Streptococcus agalactiae isolated from fish in china. Aquaculture (Amsterdam, Netherlands), 510, 84-89. https://doi.org/10.1016/j.aquaculture.2019.05.046

7. Derbise A, Aubert S, Solh NE (1997) Mapping the regions carrying the three contiguous antibiotic resistance genes aadE, sat4, and aphA-3 in the genomes of staphylococci. Antimicrobial Agents \& Chemotherapy 41: 1024-1032. https://doi.org/10.1128/aac.41.5.1024

8. Devos KM, Brown JKM, Bennetzen JL (2002) Devos KM, Brown JKM, Bennetzen JL. Genome size reduction through illegitimate recombination counteracts genome expansion in arabidopsis. Genome Res 12: 1075-1079. Genome Research 12: 1075-1079. https://doi.org/10.1101/ gr.132102

9. Doumith M, Mushtaq S, Martin V, Chaudhry A, Adkin R, Coelho J, Chalker V, Macgowan A, Woodford N, Livermore DM (2017) Genomic sequences of Streptococcus agalactiae with high-level gentamicin resistance, collected in the BSAC bacteraemia surveillance. Journal of Antimicrobial Chemotherapy 72: 2704-2707. https://doi.org/10.1093/jac/dkx207

10. Evans JJ, Bohnsack JF, Klesius PH, Whiting AA, Garcia JC, Shoemaker CA, Takahashi S (2008) Phylogenetic relationships among Streptococcus agalactiae isolated from piscine, dolphin, bovine and human sources: a dolphin and piscine lineage associated with a fish epidemic in Kuwait is also associated with human neonatal infections in Japan. Journal of Medical Microbiology 57: 13691376. https://doi.org/10.1099/jmm.0.47815-0

11. Faccone D, Lalonardi F, Abel S, Machain M, Errecalde L, Littvik A, Kauffman S, Galas M, Group WH-A, Corso A (2010) Multiple-Clones of Streptococcus agalactiae harbouring InuB gene. Journal of 
infection in developing countries 4: 580-582. https://doi.org/10.3855/jidc.941

12. Facimoto CT, Chideroli RT, Goncalves DD, Carmo AOd, Kalaphotakis E, Pereira UdP (2017) WholeGenome Sequence of Streptococcus agalactiae Strain S13, Isolated from a Fish Eye from a Nile Tilapia Farm in Southern Brazil. Genome Announcements 5: e00917-00917. https://doi.org/10.1128/genomeA.00917-17

13. Florindo C, Viegas S, Paulino A, Rodrigues E, Gomes JP, Borrego MJ (2010) Molecular characterization and antimicrobial susceptibility profiles in Streptococcus agalactiae colonizing strains: association of erythromycin resistance with subtype III-1 genetic clone family. Clinical Microbiology and Infection 16: 1458-1463. https://doi.org/10.1111/j.1469-0691.2010.03106.x

14. Godoy DT, Carvalho-Castro GA, Leal CA, Pereira UP, Leite RC, Figueiredo HC (2013) Genetic diversity and new genotyping scheme for fish pathogenic Streptococcus agalactiae. Letters in applied microbiology 57: 476-483. https://doi.org/10.1111/lam.12138

15. He EM, Chen CW, Guo Y, Hsu MH, Zhang L, Chen HL, Zhao GP, Chiu CH, Zhou Y (2017) The genome of serotype VI Streptococcus agalactiae serotype VI and comparative analysis. Gene 597: 59-65 https://doi.org/10.1016/j.gene.2016.10.030

16. Jaglarz A, Gurgul A, Leigh WJ, Costa JZ, Thompson KD (2018) Complete Genome Sequences of Three Fish-Associated Streptococcus agalactiae Isolates. Genome Announcements 6: e00025-00018. https://doi.org/10.1128/genomeA.00025-18

17. Jiang S, Park SE, Yadav P, Paoletti LC, Wessels MR (2012) Regulation and function of pilus island 1 in group B streptococcus. Journal of bacteriology 194: 2479-2490. https://doi.org/10.1128/JB.00202-12

18. Jones N, Bohnsack JF, Takahashi S, Oliver KA, Chan MS, Kunst F, Glaser P, Rusniok C, Crook DWM, Harding RM, Bisharat N, Spratt BG (2003) Multilocus Sequence Typing System for Group B Streptococcus. Journal of clinical microbiology 41: 2530-2536. https://doi.org/10.1128/jcm.41.6.2530-2536.2003

19. Kannika K, Pisuttharachai D, Srisapoome P, Wongtavatchai J, Kondo H, Hirono I, Unajak S, Areechon N (2017) Molecular serotyping, virulence gene profiling and pathogenicity of Streptococcus agalactiae isolated from tilapia farms in Thailand by multiplex PCR. Journal of applied microbiology 122: 1497-1507. https://doi.org/10.1111/jam.13447

20. Kawamura Y, Fujiwara H, Mishima N, Tanaka Y, Tanimoto A, Ikawa S, Itoh Y, Ezaki T (2003) First Streptococcus agalactiae Isolates Highly Resistant to Quinolones, with Point Mutations in gyrA and parC. Antimicrobial Agents \& Chemotherapy 47: 3605-3609. https://doi.org/10.1128/AAC.47.11.3605-3609.2003

21. Kayansamruaj P, Pirarat N, Katagiri T, Hirono I, Rodkhum C (2014) Molecular characterization and virulence gene profiling of pathogenic Streptococcus agalactiae populations from tilapia (Oreochromis sp.) farms in Thailand. Journal of veterinary diagnostic investigation: official publication of the American Association of Veterinary Laboratory Diagnosticians, Inc 26: 488-495. https://doi.org/10.1177/1040638714534237 
22. Lee GC, Long SW, Musser JM, Beres SB, Olsen RJ, Dallas SD, Nunez YO, Frei CR (2015) Comparative whole genome sequencing of community-associated methicillin-resistant Staphylococcus aureus sequence type 8 from primary care clinics in a Texas community. Pharmacotherapy 35: 220-228. https://doi.org/10.1002/phar.1536

23. Li L, Wang R, Liang W, Gan X, Huang T, Huang Y, Li J, Shi Y, Chen M, Luo H (2013) Rare serotype occurrence and PFGE genotypic diversity of Streptococcus agalactiae isolated from tilapia in China. Veterinary microbiology 167: 719-724. https://doi.org/10.1016/j.vetmic.2013.09.001

24. Liu G, Zhang W, Lu C (2012) Complete genome sequence of Streptococcus agalactiae GD201008001, isolated in China from tilapia with meningoencephalitis. Journal of bacteriology 194: 6653. https://doi.org/10.1128/JB.01788-12

25. Liu G, Zhang W, Lu C (2013) Comparative genomics analysis of Streptococcus agalactiae reveals that isolates from cultured tilapia in China are closely related to the human strain A909. Bmc Genomics 14: 775-785. https://doi.org/10.1186/1471-2164-14-775

26. Lynch M (2006) Streamlining and simplification of microbial genome architecture. Annual review of microbiology 60: 327-349. https://doi.org/10.1146/annurev.micro.60.080805.142300

27. Nobbs AH, Rosini R, Rinaudo CD, Maione D, Grandi G, Telford JL (2008) Sortase A utilizes an ancillary protein anchor for efficient cell wall anchoring of pili in Streptococcus agalactiae. Infection and immunity 76: 3550-3560. https://doi.org/10.1128/IAI.01613-07

28. Pereira UP, Mian GF, Oliveira IC, Benchetrit LC, Costa GM, Figueiredo HC (2010) Genotyping of Streptococcus agalactiae strains isolated from fish, human and cattle and their virulence potential in Nile tilapia. Veterinary microbiology 140: 186-192. https://doi.org/10.1016/j.vetmic.2009.07.025

29. Poyart C, Jardy L, Quesne G, Berche P, Trieu-Cuot P (2003) Genetic basis of antibiotic resistance in Streptococcus agalactiae strains isolated in a French hospital. Antimicrobial agents and chemotherapy 47: 794-797. https://doi.org/10.1128/aac.47.2.794-797.2003

30. Pridgeon JW, Zhang D (2014) Complete Genome Sequence of a Virulent Streptococcus agalactiae Strain, 138P, Isolated from Diseased Nile Tilapia. Genome Announcements 2: e00295-00296. https://doi.org/10.1128/genomea.00295-14

31. Rajendram P, Mar Kyaw W, Leo YS, Ho H, Chen WK, Lin R, Pratim P, Badaruddin H, Ang B, Barkham T, Chow A (2016) Group B Streptococcus Sequence Type 283 Disease Linked to Consumption of Raw Fish, Singapore. Emerging infectious diseases 22: 1974-1977. https://doi.org/10.3201/eid2211.160252

32. Ren SY, Geng Y, Wang KY, Zhou ZY, Lai WM (2013) Streptococcus agalactiae Infection in Domestic Rabbits, Oryctolagus cuniculus. Transboundary \& Emerging Diseases 61: e92-95. https://doi.org/10.1111/tbed.12073

33. Rinaudo CD, Rosini R, Galeotti CL, Berti F, Necchi F, Reguzzi V, Ghezzo C, Telford JL, Grandi G, Maione D (2010) Specific involvement of pilus type 2a in biofilm formation in group B Streptococcus. PloS one 5: e9216. https://doi.org/10.1371/journal.pone.0009216 
34. Rosini R, Rinaudo CD, Soriani M, Lauer P, Mora M, Maione D, Taddei A, Santi I, Ghezzo C, Brettoni C, Buccato S, Margarit I, Grandi G, Telford JL (2006) Identification of novel genomic islands coding for antigenic pilus-like structures in Streptococcus agalactiae. Molecular microbiology 61: 126-141. https://doi.org/10.1111/j.1365-2958.2006.05225.x

35. Salma R, Dabboussi F, Khudary R, Hamze M (2013) gyrA and parC mutations in quinolone-resistant clinical isolates of Pseudomonas aeruginosa from Nini Hospital in north Lebanon. Journal of Infection \& Chemotherapy 19: 77-81. https://doi.org/10.1007/s10156-012-0455-y

36. Siguier P, Gourbeyre E, Chandler M (2014) Bacterial insertion sequences: their genomic impact and diversity. FEMS microbiology reviews 38: 865-891. https://doi.org/10.1111/1574-6976.12067

37. Slotved HC, Kong F, Lambertsen L, Sauer S, Gilbert GL (2007) Serotype IX, a Proposed New Streptococcus agalactiae Serotype. Journal of clinical microbiology 45: 2929-2936. https://doi.org/10.1128/JCM.00117-07

38. Veeraraghavan B, Devanga Ragupathi NK, Santhanam S, Verghese VP, Inbanathan FY, Livingston C (2017) Whole genome shotgun sequencing of Indian strains of Streptococcus agalactiae. Genomics data 14: 63-65. https://doi.org/10.1016/j.gdata.2017.10.001

39. Wang R, Li L, Huang Y, Huang T, Tang J, Xie T, Lei A, Luo F, Li J, Huang Y, Shi Y, Wang D, Chen M, Mi Q, Huang W (2017) Pathogenicity of Human ST23 Streptococcus agalactiae to Fish and Genomic Comparison of Pathogenic and Non-pathogenic Isolates. Frontiers in microbiology 8: 1933. https://doi.org/10.3389/fmicb.2017.01933

40. Werner G, Hildebrandt B, Witte W (2003) Linkage of erm(B) and aadE-sat4-aphA-3 in MultipleResistant Enterococcus faecium Isolates of Different Ecological Origins. Microbial Drug Resistance 9 Suppl 1: S9-16. https://doi.org/10.1089/107662903322541847

41. Zankari E, Hasman H, Cosentino S, Vestergaard M, Rasmussen S, Lund O, Aarestrup FM, Larsen MV (2012) Identification of acquired antimicrobial resistance genes. The Journal of antimicrobial chemotherapy 67: 2640-2644. https://doi.org/10.1093/jac/dks261

42. Zhang Xin-yan, Fan Hai-ping, Zhong Quan-fu, Zhuo Yu-chen, Lin Yu, Zeng Zhan-zhuang (2008) Isolation, identification and pathogenicity of Streptococcus agalactiae from tilapia. Journal of Fisheries of China 32: $772-779$ (in Chinese). doi 10.3724/sp.j.00001

43. Zhao S, Tyson GH, Chen Y, Li C, Mukherjee S, Young S, Lam C, Folster JP, Whichard JM, McDermott PF (2016) Whole-Genome Sequencing Analysis Accurately Predicts Antimicrobial Resistance Phenotypes in Campylobacter spp. Applied and environmental microbiology 82: 459-466. https://doi.org/10.1128/AEM.02873-15

\section{Figures}


a

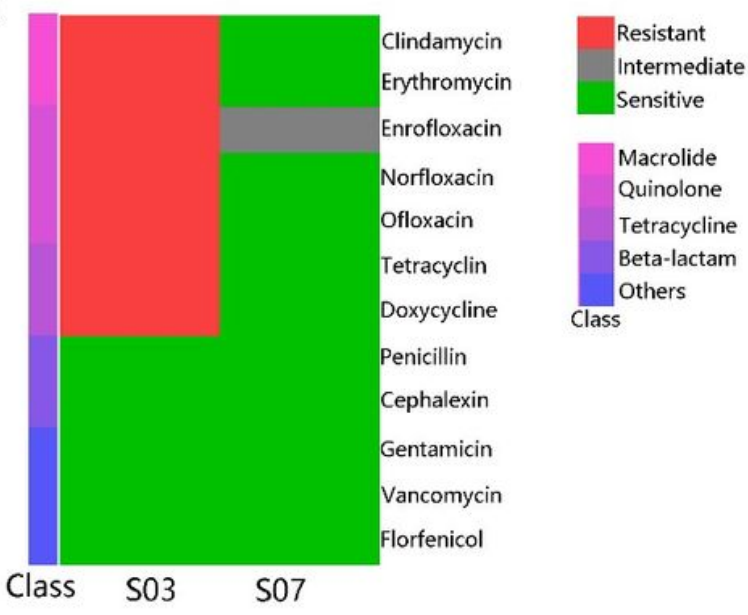

b

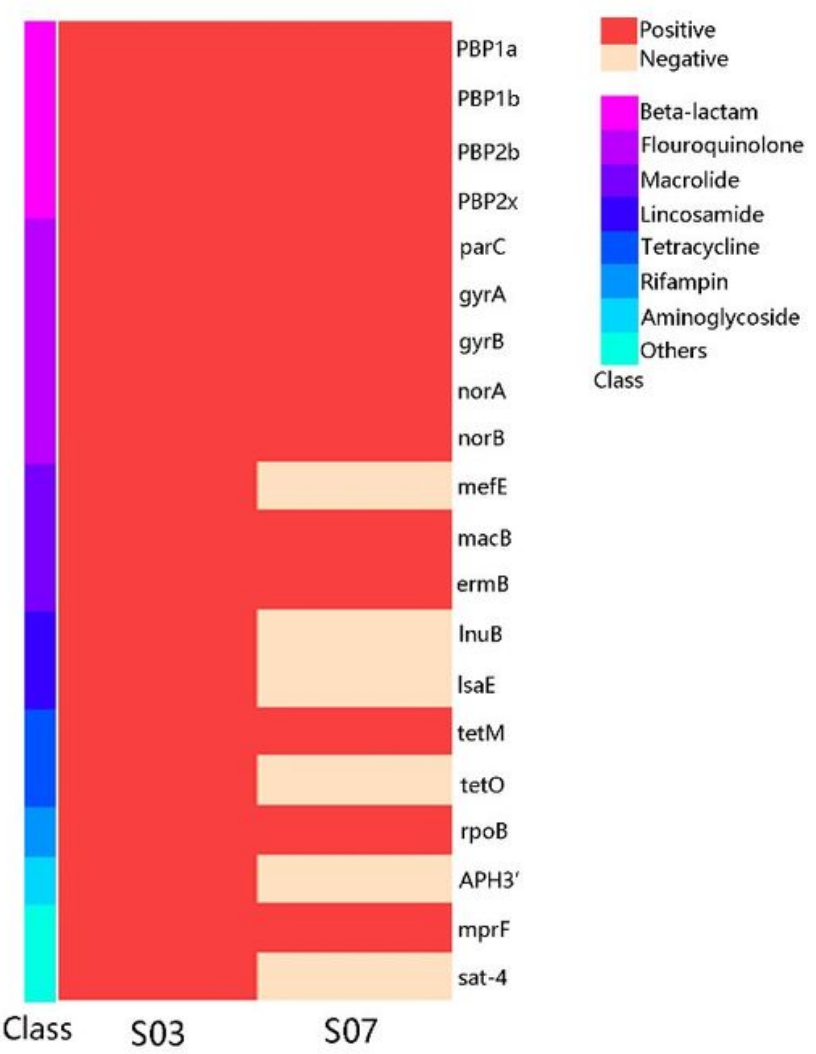

\section{Figure 1}

Heat maps of antibiotic resistance results (a) and resistance genes (b) of GBS S03 and S07, generated using graphpad (version 8.0.1). 


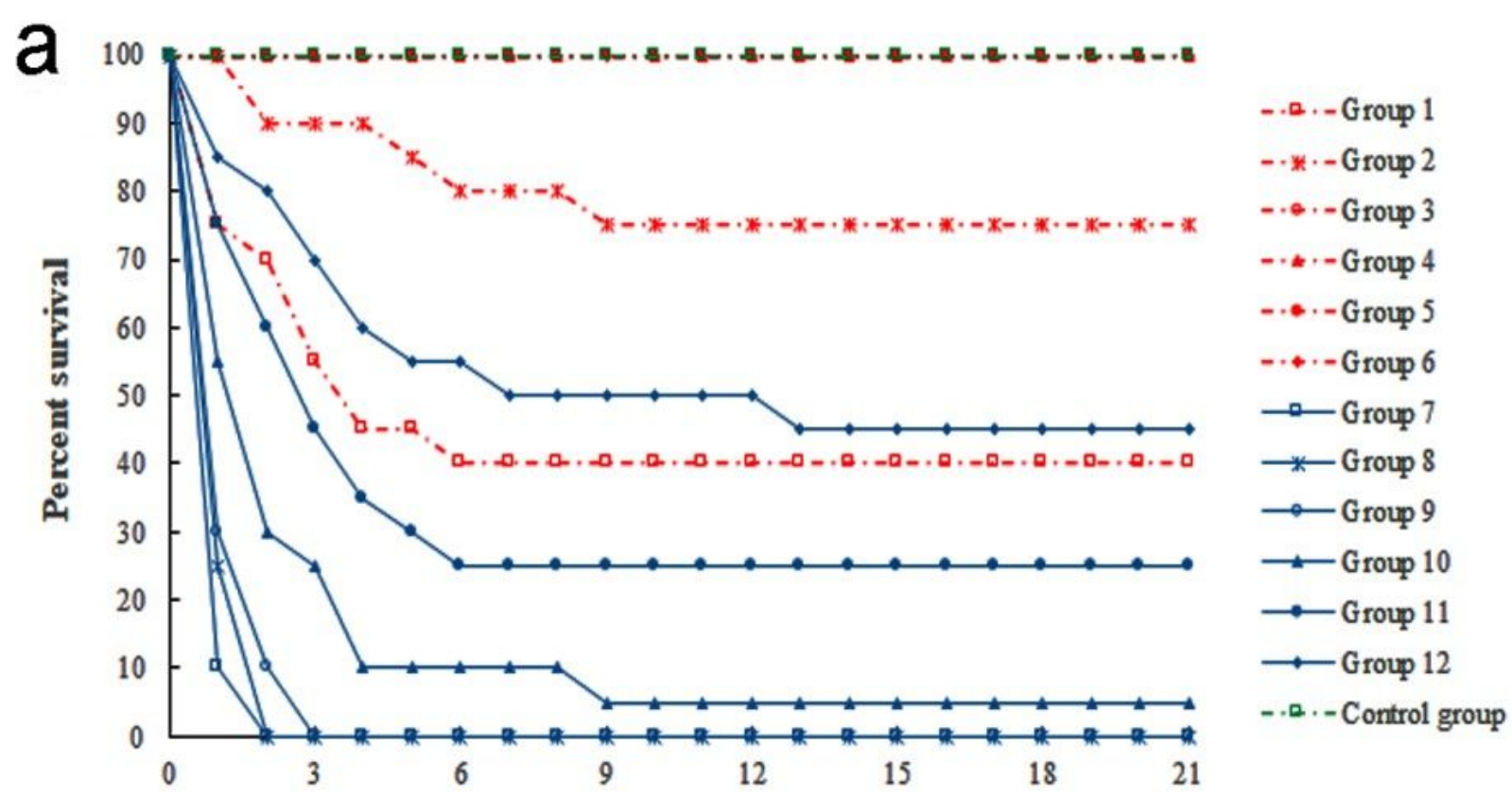

Day post-challenge

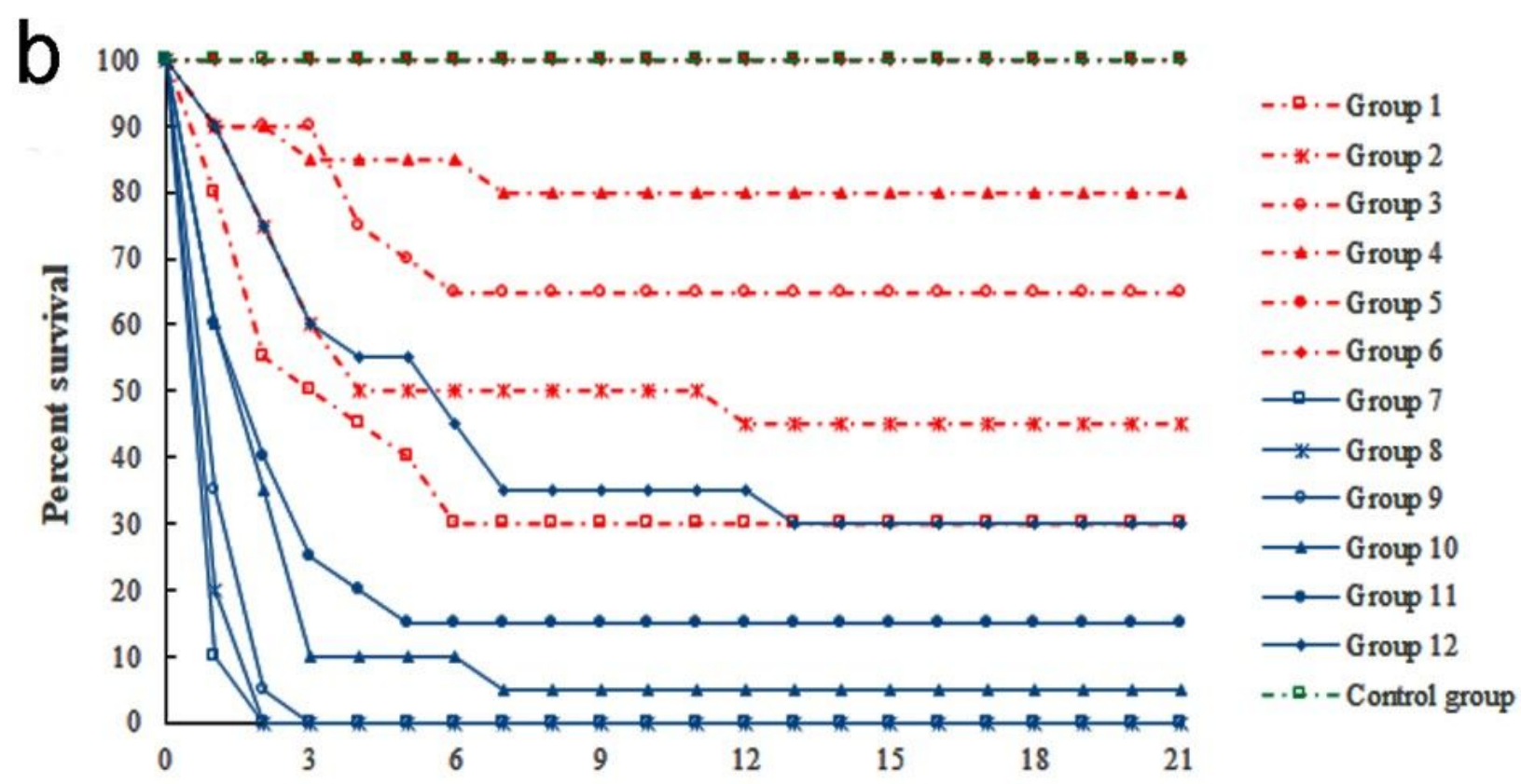

Day post-challenge

\section{Figure 2}

Percent survival (\%) of Schizothorax prenanti (a) and Danio rerio (b) after challenge with different concentrations of GBS S03 and S07. Group 1-6: survival curves of fish injected with S03 suspensions at $1.0 \times 109,1.0 \times 108,1.0 \times 107,1.0 \times 106,1.0 \times 105,1.0 \times 104 \mathrm{cfu} \cdot \mathrm{ml}-1$ concentrations respectively; Group 7-12: survival curves of fish injected with S07 suspensions at 1.0×109, 1.0×108, 1.0×107, 1.0×106, 1.0×105, 
$1.0 \times 104 \mathrm{cfu} \cdot \mathrm{ml}-1$ concentrations respectively; Control group: survival curves of fish injected with $0.8 \%$ $\mathrm{NaCl}$ solution.

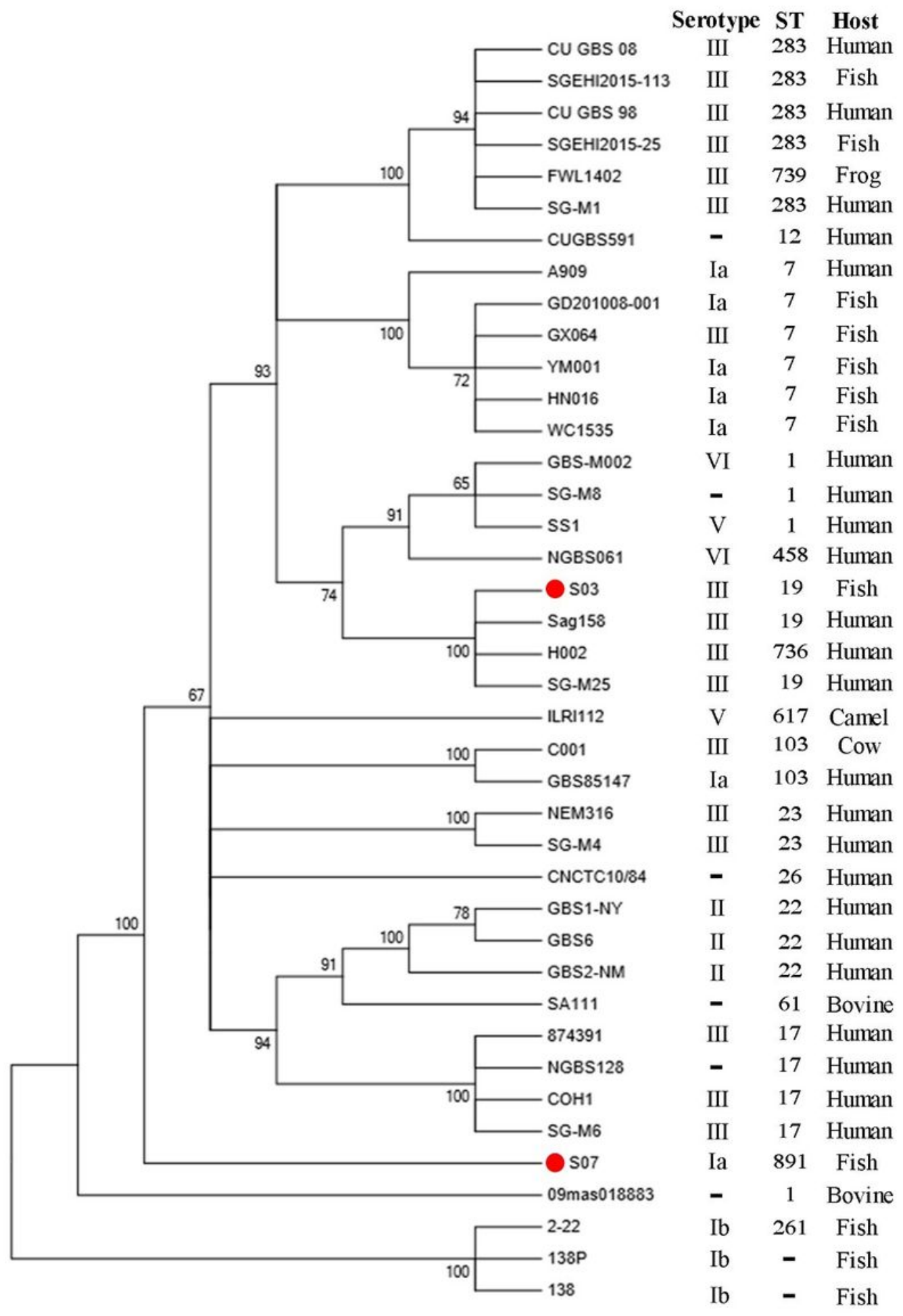

Figure 3

Molecular phylogenetic tree of the evolutionary relationship among different GBS strains using the Neighbor-Joining algorithm and 1000 bootstraps. 


\section{Supplementary Files}

This is a list of supplementary files associated with this preprint. Click to download.

- Supplementarymaterial.docx 